\title{
Shear Current Effects on Monochromatic Water Waves Crossing Trenches
}

\author{
Jun-Whan Lee, ${ }^{1}$ Koo-Yong Park, ${ }^{2}$ and Yong-Sik Cho' \\ ${ }^{1}$ Department of Civil and Environmental Engineering, Hanyang University, 222 Wangsimni-ro, Seongdong-gu, \\ Seoul 133-791, Republic of Korea \\ ${ }^{2}$ Civil and Environment Division, Hyundai Engineering and Construction, Gye-dong, Jongno-gu, Seoul 110-920, Republic of Korea
}

Correspondence should be addressed to Yong-Sik Cho; ysc59@hanyang.ac.kr

Received 28 October 2014; Accepted 6 January 2015

Academic Editor: Michael Meylan

Copyright (C) 2015 Jun-Whan Lee et al. This is an open access article distributed under the Creative Commons Attribution License, which permits unrestricted use, distribution, and reproduction in any medium, provided the original work is properly cited.

\begin{abstract}
The reflection coefficients of monochromatic water waves over trenches with shear current are estimated analytically. The diffraction of waves by an abrupt depth change and shear current is formulated by the matched eigenfunction expansion method. The proper number of steps and evanescent modes are proposed by a series of convergence tests. The accuracy of the predicted reflection coefficients is checked by estimating the wave energy. Reflection and transmission characteristics are studied for various shear current conditions. The different combinations of strength, width of shear current, and incident wave angle with constant water depth topography are examined. The optimal figure of the trench with shear current is obtained by estimating the reflection coefficients for various sloped transitions. The resonant reflection of the water waves is found by multiarrayed optimal trenches and the interaction of sinusoidally varying topography with shear current.
\end{abstract}

\section{Introduction}

When water waves propagate over the nonuniform bottom topography and shear current field, they undergo many physical phenomenon including refraction and partial reflection. It is well known that the nearshore and coastal environments with the stability of coastal structures are significantly affected by water waves [1]. Therefore, the prediction of wave transformation is very important for coastal engineers to design counter facilities that can protect coastal structures from wave attacks. A number of wave-scattering theoretical models treating bathymetry and shear current or both have been developed.

A widely studied wave diffraction problem is abrupt depth changes. Specifically, the analytic method based on the potential flow theory has been used for decades because of its simple but absolutely accurate characteristics. By employing the well-known semianalytic matched eigenfunction expansion method to the potential flow theory, breakwaters and trenches can be represented by a series of steps. One of the earliest studies solving the scattering problem of normal incident wave over discontinuous bathymetry was given by Takano [2]. The linear integral equations were set up by the matching boundary conditions and were solved numerically for a truncated series. Miles [3] used a planewave and variational approximations and solved a scattering matrix for the case of discontinuity in depth. Kirby and Dalrymple [4] extended the previous studies to involve the obliquely incident water waves and asymmetric geometry of a rectangular trench. By formulating the scattering problem in terms of Schwinger's type integrals, Lassiter III [5] has solved the wave diffraction due to a trench containing two different fluids. Bender and Dean [6] have considered scattering of normally incident waves over a trench with sloping sides by using three different methods and concluded that the step method is valid for an arbitrary water depth, while other methods are limited to the shallow water region. Recently, several kinds of techniques for solving the scattering problem, such as Galerkin method, the first and second kinds of Bessel functions, perturbation method, have been employed [7-9]. 
Also, several kinds of conditions that cause the scattering problem, such as porous plates, floating plate, and cylinders, have been studied [10-12].

Horizontally sheared currents such as those formed at river mouths, tidal inlets, and tidal races and around coastal structures are also one of the main factors of wave transformation [13]. However, even within the linear theory framework, the analysis of the water waves across a shear current is remarkably intractable. Therefore, many approximate methods based on mild-slope, mild-shear equations, and vortex sheets have been developed. The case of incident waves on a single vortex sheet representing a shear current was studied by Evans [14] using a Galerkin approximation and was extended to a two vortex sheets problem based on conservation of wave action and vertical averaging by Smith [15]. Smith [16] also presented a variety of approximate solutions to the wave diffraction problem caused by a uniform shear current and depth change. Kirby et al. [17] developed a matched eigenfunction expansion model to study the effect of shear current flowing along a trench in finite water depth, and the model was verified with a boundary-integral-equation method. Liu et al. [18] applied the eigenfunction expansion method to the scattering problem of obliquely incident wave groups over a trench with a shear current. An arbitrary number of discontinuities of shear current were used by McKee [19] to allow realistic representation of natural shear current by gradual transitions in water of a constant depth. The results were compared with the mild-shear equation model [20] and the extended mild-shear equation model [21].

One of the most interesting topics in the wave scattering problem is Bragg resonant reflection. When the bottom topography contains the patches of periodic undulations and the wavelength of the bottom ripple is one-half of the wavelength of the incident wave, the so called Bragg conditions, waves are scattered and amplified due to the resonant effect [22]. Dalrymple and Kirby [23] used a boundary integral equation method and calculated the wavenumber ratio for the obliquely incident wave that could yield the resonant reflection. Guazzelli et al. [24] compared the experimental measurements with numerical predictions obtained from the potential theory of linear waves. McKee [25] investigated the Bragg reflection by sinusoidally varying the shear current using a mild-shear equation and compared the result with the modulation theory. The model based on the eigenfunction expansion method was developed by Cho and Lee [26] and the obtained results were compared with the laboratory measurements of singly and doubly sinusoidally varying topographies. For a practical counter facility's design using the Bragg reflection concept, Kirby and Anton [27] and Hsu et al. [28] investigated periodically spaced rectified sinusoidal and rectangular breakwaters, respectively. Although multiarrayed breakwaters show good performance in wave protection, these are not suitable for the region where vessel seaway is required. For this problem, Kim et al. [29] studied the reflection by multiarrayed trenches and suggested the optimal figure of trench which reflects the incident wave mostly. However, the proper number of steps and evanescent modes are not proposed, and the effect of shear current is not considered.

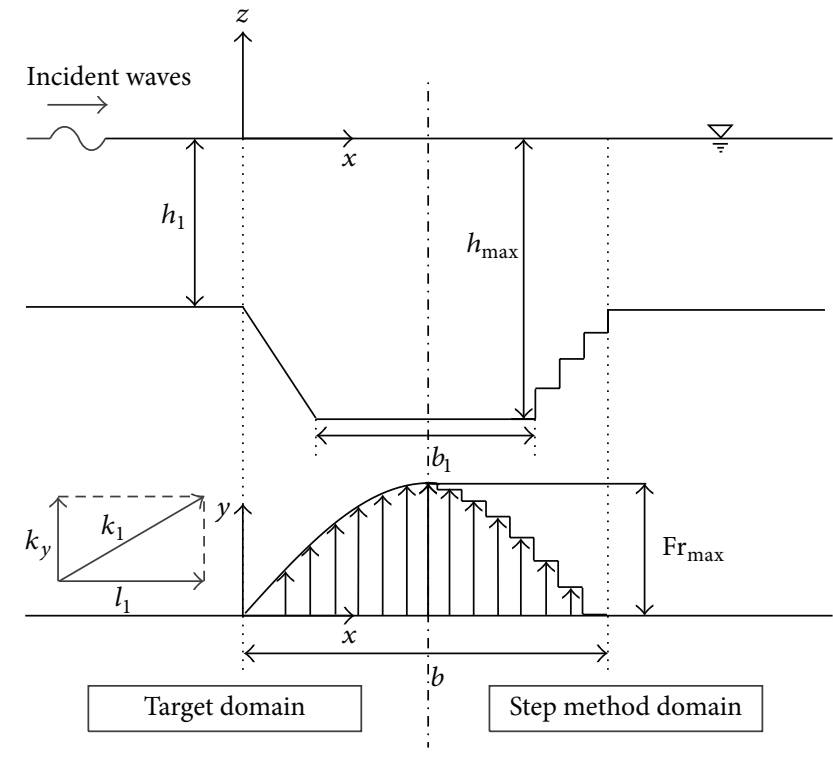

FIgURE 1: Definition sketch of a computational domain.

The aim of the present study is to extend Cho and Lee [30] by including shear current effects and to investigate the role of shear current for several cases. In this study, the semianalytic matched eigenfunction expansion technique is used to calculate the reflection coefficients. Preceding the investigation of shear current effects, after explaining the methodology briefly for completeness, the proper number of steps and evanescent modes are suggested to fully converge. Next, the significance of shear current in the wave diffraction problem is checked by adding several shear currents. The investigation is carried out for finding the main factors of the maximum reflection coefficient and its location, as well as finding the optimal figure of trench. Case studies for ideal conditions are conducted to identify the resonant re-flection.

\section{Eigenfunction Expansion Method}

The diffraction of monochromatic waves by an abrupt depth change and shear current is formulated by the eigenfunction expansion method. As shown in Figure 1, the variation of the bottom topography is limited to the $x$-axis direction and shear current occurs only in the $y$-axis direction. The origin of the coordinate system is placed at the beginning point where water depth or shear current differs from the incident condition. The regions where the shear current and the bottom topography change are represented by a finite number of steps; thus, the parameters are constant at each region. The relative strength of the shear current is represented by the Froude number as

$$
\operatorname{Fr}=\frac{V}{\sqrt{g h}},
$$

where $V$ is the shear current velocity, $g$ is the gravity acceleration, and $h$ is the water depth. By dividing the domain into several steps and assuming that the shear current 
is small enough so that there would be no possibility of turbulence flow, we could extend the scope of analysis to various topographies with nonhomogeneous shear current $[15,31]$.

Following Cho and Lee [30], the velocity potential defined from incompressible and irrotational flow assumption satisfies the Laplace equation. Solving the Laplace equation with boundary conditions at each discontinuity, the velocity potential functions of the monochromatic waves within each region can be written as the combination of the propagating mode and the evanescent modes like (2). The propagating mode varies sinusoidally, and the evanescent modes are the standing waves that decay exponentially in a horizontal direction:

$$
\begin{aligned}
& \Phi_{m}^{R}=\left[\begin{array}{c}
A_{m}^{R} \exp \left(+i l_{m} x\right) \cosh k_{m}\left(h_{m}+z\right) \\
+\sum_{n=1}^{\infty} B_{m, n}^{R} \exp \left(-\lambda_{m, n} x\right) \cos K_{m, n}\left(h_{m}+z\right)
\end{array}\right] \\
& \text { - } \exp \left[i\left(k_{y} y-\omega t\right)\right] \text {, } \\
& \Phi_{m}^{L}=\left[\begin{array}{c}
A_{m}^{L} \exp \left(-i l_{m} x\right) \cosh k_{m}\left(h_{m}+z\right) \\
+\sum_{n=1}^{\infty} B_{m, n}^{L} \exp \left(+\lambda_{m, n} x\right) \cos K_{m, n}\left(h_{m}+z\right)
\end{array}\right] \\
& \text { - } \exp \left[i\left(k_{y} y-\omega t\right)\right] \text {, }
\end{aligned}
$$

where superscripts $R$ and $L$ represent the right and left propagating directions, while subscripts $m$ and $n$ denote the region number and the evanescent modes, respectively. $A_{m}^{R}$, $B_{m, n}^{R}, A_{m}^{L}$, and $B_{m, n}^{L}$ are the complex amplitude functions, $h_{m}$ is the water depth in the $m$ th region, $z$ is the vertical coordinate, and $\omega$ is the angular frequency of the wave.

The $x$-axis direction wave number of the propagating mode and the evanescent modes $l_{m}, \lambda_{m, n}$ are calculated, respectively, by

$$
l_{m}=\left[k_{m}^{2}-k_{y}^{2}\right]^{0.5}, \quad \lambda_{m, n}=\left[K_{m, n}^{2}+k_{y}^{2}\right]^{0.5},
$$

where $k_{y}$ is the $y$-axis direction wavenumber component and the propagating mode and evanescent modes wavenumbers $k_{m}, K_{m, n}$ are the real roots of the dispersion relations at the $m$ th region. To consider the shear current effect, the intrinsic angular frequency (or Doppler-shifted frequency) $\sigma_{m}$ and dispersion relations are defined as follows:

$$
\begin{gathered}
\sigma_{m}^{2}=\left(\omega-V_{m} k_{y}\right)^{2}=g k_{m} \tanh k_{m} h_{m}, \\
\sigma_{m}^{2}=\left(\omega-V_{m} k_{y}\right)^{2}=-g K_{m, n} \tan K_{m, n} h_{m},
\end{gathered}
$$

where $V_{m}$ is the shear current velocity of $m$ th region. In this study, only considering $\sigma_{m}>0, k_{m}>k_{y}$ condition for the first region where the restricted condition is identical to Kirby et al. [17].

To calculate the complex amplitude functions, there should be two matching conditions at the junctions where the two steps meet. Two conditions are expressed as (5), which are the continuity of the horizontal flux in the $x$-axis direction and the continuity of pressure at each depth discontinuity that is necessary to avoid infinite accelerations of the fluid particles on the vortex sheet [19]. Detailed derivation of the matching conditions has been reviewed in Evans [14] and Kirby et al. [17]:

$$
\begin{aligned}
& \frac{1}{\sigma_{m}} \frac{\partial \Phi_{m}}{\partial x}=\frac{1}{\sigma_{m+1}} \frac{\partial \Phi_{m+1}}{\partial x}, \quad x=x_{m}, \\
& \max \left(-h_{m},-h_{m+1}\right) \leq z \leq 0, \\
& \sigma_{m} \Phi_{m}=\sigma_{m+1} \Phi_{m+1}, \quad x=x_{m}, \\
& \max \left(-h_{m},-h_{m+1}\right) \leq z \leq 0 .
\end{aligned}
$$

By putting (2) to two matching conditions and integrating them from bottom to top by using orthogonality, we could solve the linear matrix equations numerically. Here, using the boundary condition whose normal velocity on the rigid wall is zero, the integral range was categorized into two cases.

$$
\begin{aligned}
& \text { Case } 1\left(x=x_{m},-h_{m}>-h_{m+1}\right) \text {. Consider } \\
& \qquad \begin{array}{l}
\int_{-h_{m}}^{0} \frac{1}{\sigma_{m}} \frac{\partial \Phi_{m}}{\partial x} \cosh \left[k_{m+1}\left(h_{m+1}+z\right)\right] d z \\
=\int_{-h_{m+1}}^{0} \frac{1}{\sigma_{m+1}} \frac{\partial \Phi_{m+1}}{\partial x} \cosh \left[k_{m+1}\left(h_{m+1}+z\right)\right] d z \\
\int_{-h_{m}}^{0} \sigma_{m} \Phi_{m} \cosh \left[k_{m}\left(h_{m}+z\right)\right] d z \\
=\int_{-h_{m}}^{0} \frac{\sigma_{m+1} \Phi_{m+1} \cosh \left[k_{m}\left(h_{m}+z\right)\right] d z}{\int_{-h_{m}}^{0}} \frac{1}{\sigma_{m}} \frac{\partial \Phi_{m}}{\partial x} \cos \left[K_{m+1, n}\left(h_{m+1}+z\right)\right] d z \\
=\int_{-h_{m+1}}^{0} \frac{1}{\sigma_{m+1}} \frac{\partial \Phi_{m+1}}{\partial x} \cos \left[K_{m+1, n}\left(h_{m+1}+z\right)\right] d z \\
\int_{-h_{m}}^{0} \sigma_{m} \Phi_{m} \cos \left[K_{m, n}\left(h_{m}+z\right)\right] d z \\
=\int_{-h_{m}}^{0} \sigma_{m+1} \Phi_{m+1} \cos \left[K_{m, n}\left(h_{m}+z\right)\right] d z .
\end{array}
\end{aligned}
$$

Case $2\left(x=x_{m},-h_{m}<-h_{m+1}\right)$. Consider

$$
\begin{aligned}
& \int_{-h_{m}}^{0} \frac{1}{\sigma_{m}} \frac{\partial \Phi_{m}}{\partial x} \cosh \left[k_{m}\left(h_{m}+z\right)\right] d z \\
& \quad=\int_{-h_{m+1}}^{0} \frac{1}{\sigma_{m+1}} \frac{\partial \Phi_{m+1}}{\partial x} \cosh \left[k_{m}\left(h_{m}+z\right)\right] d z
\end{aligned}
$$




$$
\begin{aligned}
& \int_{-h_{m+1}}^{0} \sigma_{m} \Phi_{m} \cosh \left[k_{m+1}\left(h_{m+1}+z\right)\right] d z \\
& \quad=\int_{-h_{m+1}}^{0} \sigma_{m+1} \Phi_{m+1} \cosh \left[k_{m+1}\left(h_{m+1}+z\right)\right] d z \\
& \int_{-h_{m}}^{0} \frac{1}{\sigma_{m}} \frac{\partial \Phi_{m}}{\partial x} \cos \left[K_{m, n}\left(h_{m}+z\right)\right] d z \\
& =\int_{-h_{m+1}}^{0} \frac{1}{\sigma_{m+1}} \frac{\partial \Phi_{m+1}}{\partial x} \cos \left[K_{m, n}\left(h_{m}+z\right)\right] d z \\
& \int_{-h_{m+1}}^{0} \sigma_{m} \Phi_{m} \cos \left[K_{m+1, n}\left(h_{m+1}+z\right)\right] d z \\
& =\int_{-h_{m+1}}^{0} \sigma_{m+1} \Phi_{m+1} \cos \left[K_{m+1, n}\left(h_{m+1}+z\right)\right] d z .
\end{aligned}
$$

Because there are two matching conditions with $M-1$ step junctions and $N+1$ modes consist of one propagating mode and $N$ evanescent modes, linear matrix that has $2(M-$ 1) $(N+1)$ unknown could be derived where $M$ and $N$ are the number of total steps and evanescent modes, respectively. The complex amplitude functions were calculated by Gauss elimination after LU factorization in this study. The absolute values of the reflected amplitude of the first region and the transmitted amplitude of the last region derive from the free surface boundary condition as follows:

$$
\begin{gathered}
a_{L}=g^{-1}\left|A_{1}^{L}\right| \sigma_{1} \cosh k_{1} h_{1}, \\
a_{R}=g^{-1}\left|A_{M}^{R}\right| \sigma_{M} \cosh k_{M} h_{M} .
\end{gathered}
$$

By using the absolute value of the right-going initial complex amplitude function as one $\left(\left|A_{1}^{R}\right|=1\right)$, a reflection coefficient $(R)$ and a transmission coefficient $(T)$ are simply calculated by using

$$
R=\left|A_{1}^{L}\right|, \quad T=\frac{\sigma_{M}}{\sigma_{1}} \frac{\cosh k_{M} h_{M}}{\cosh k_{1} h_{1}}\left|A_{M}^{R}\right| .
$$

In order to verify the values of the reflection and transmission coefficients calculated by the method above, the conservation of energy (also called the wave action flux) consideration represented in (10) is applied $[17,26]$ :

$$
R^{2}+\frac{n_{j} k_{1} \cos \theta_{j}}{n_{1} k_{j} \cos \theta_{1}} T^{2}=1, \quad n_{j}=\frac{1}{2}\left[1+\frac{2 k_{j} h_{j}}{\sinh 2 k_{j} h_{j}}\right] \text {. }
$$

\section{Numerical Results and Discussions}

In this section, before investigating the resonant reflection caused by trenches and shear currents, we investigate the characteristics of the unity shear current and trench. First, we show the role of the shear current by absenting or changing the shear current, while keeping the bathymetry. To find the characteristics of the reflection coefficient due to the shear current, many combinations of the strength,

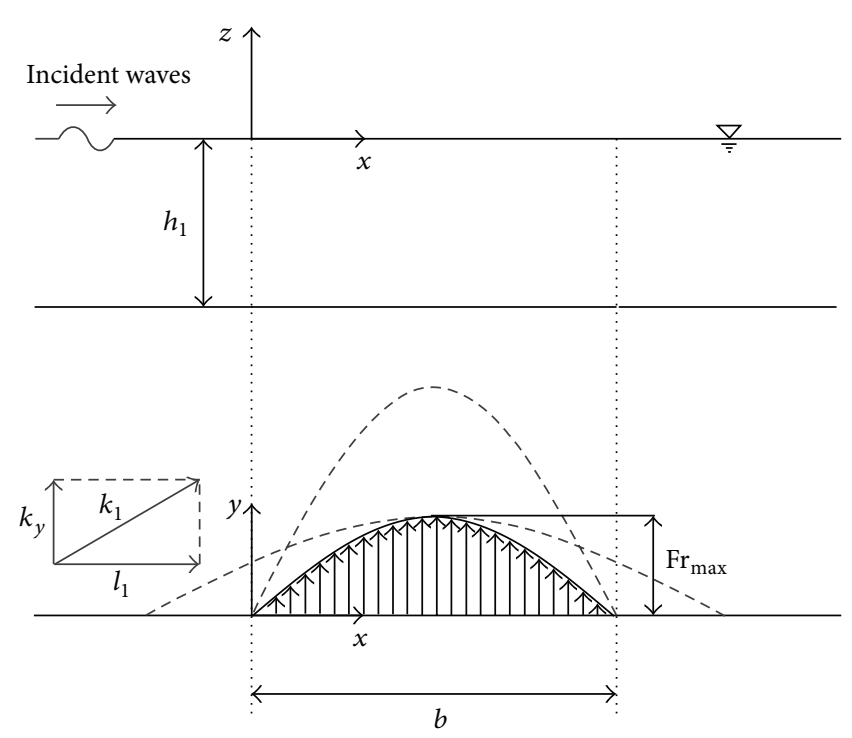

FIGURE 2: A schematic sketch of constant water depth with sinusoidal shape shear current.

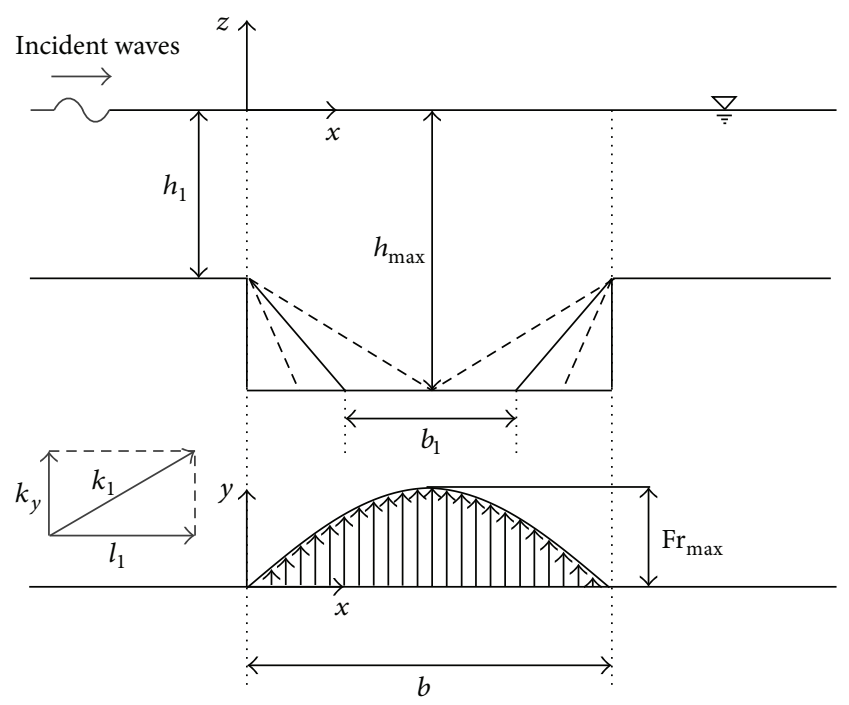

FIGURE 3: A schematic sketch of a symmetric trapezoidal trench with sinusoidal shape shear current.

width of the shear current, and incident wave angle with uniform depth topography were generated (see Figure 2). Also, by changing the slope of the symmetric and asymmetric trapezoidal trench, the optimal figure of the trench where the shear current flows along the trench are suggested (see Figures 3 and 4). Although the step method is valid in the arbitrary water depth, following Bender and Dean [6], we limit the investigating domains from the shallow water to the intermediate water regions to visualize the figures.

3.1. Convergence Test for Steps and Evanescent Modes. The sinusoidal shape shear current was used in this study to represent the natural shear current which has maximum 


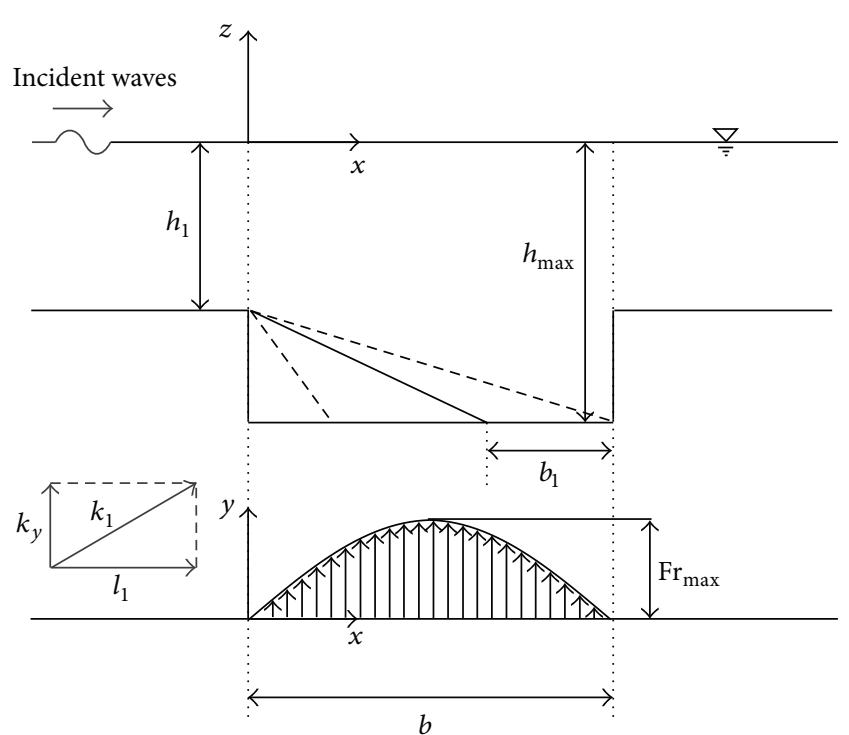

FIGURE 4: A schematic sketch of an asymmetric trapezoidal trench with sinusoidal shape shear current.

velocity at the center of the shear current. The shear current profile is of the form in

$$
\operatorname{Fr}(x)=\operatorname{Fr}_{\max } \sin \left(\frac{x}{b} \pi\right), \quad 0 \leq x \leq b .
$$

Preceding the analysis of the reflection coefficient, a proper number of steps and evanescent modes should be determined by a convergence test. This is significant because the accuracy of the solution cannot be guaranteed if the number of steps and evanescent modes are not enough to converge. Otherwise, selecting the overestimated number of steps and evanescent modes, the total running time will increase drastically.

As shown in Figure 5, two cases were conducted to get an accurate solution for the propagating mode which refers to the plane wave approximate condition. We only used an odd number of steps to make a symmetric and asymmetric trench and shear current.

Case 1. Trapezoidal trench without shear current: Incident wave angle $\theta=30^{\circ}$, dimensionless top width of a trench $b / h_{1}=10$, dimensionless bottom width of a trench $b_{1} / h_{1}=0$, and dimensionless depth of a trench $h_{\max } / h_{1}=2$ were used.

Case 2. Constant water depth with sinusoidal shape shear current: Incident wave angle $\theta=30^{\circ}$, dimensionless width of shear current $b / h_{1}=10$, and maximum Froude number $\mathrm{Fr}_{\max }=0.2$ were used.

Bender and Dean [6] used one step for the abrupt transition trenches and ten steps for all the other slopes. However, in this study, based on a convergence test, 27 steps of one side slope $\left(m^{\prime}=(m-1) / 2\right)$ for the mild slope symmetric trench $\left(\left(h_{\max }-h_{1}\right) /\left(b-b_{1}\right)<0.5\right)$ and a gradually decreasing number of steps for the steep slope symmetric trench $\left(\left(h_{\max }-h_{1}\right) /\left(b-b_{1}\right)>0.5\right)$ were used in the slope

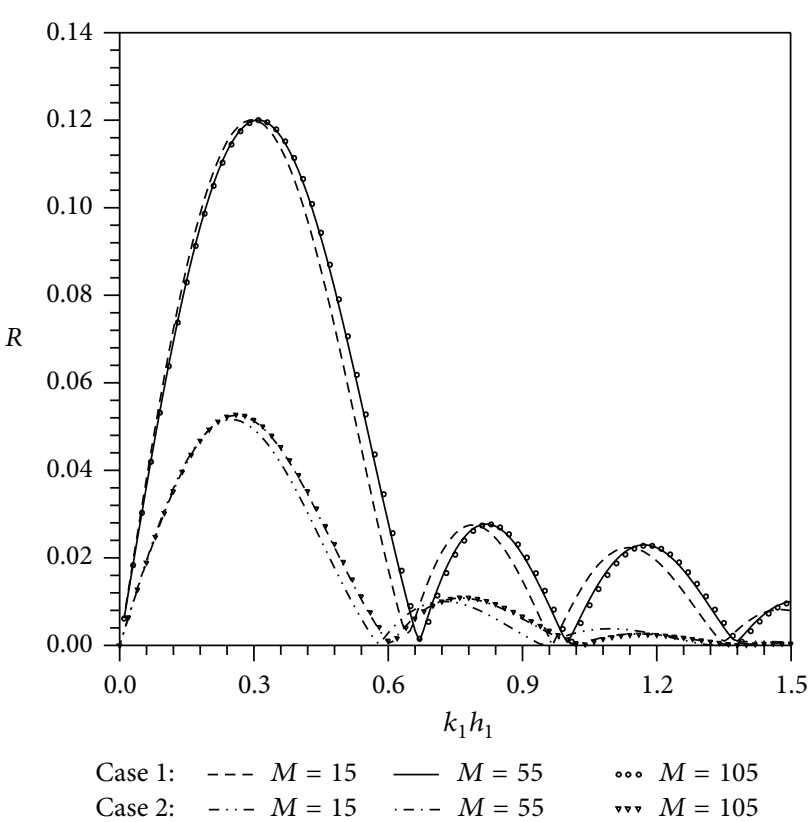

FIgURE 5: A convergence test for different number of steps.

approximation. Double steps were used for the asymmetric trench.

The number of evanescent modes must also be large enough to ensure the convergence of the solution. To visualize the role of evanescent modes in an abrupt depth change, another case was conducted.

Case 3. A rectangular trench with sinusoidal shape shear current: Incident wave angle $\theta=30^{\circ}$, dimensionless width of trench and shear current $b / h_{1}=10$, dimensionless depth of trench $h_{\max } / h_{1}=2$, maximum Froude number $\mathrm{Fr}_{\max }=$ 0.15708 , and the number of steps $M=55$ were used.

Figure 6 shows the variation of the reflection coefficient $R$ of Cases 2 and 3 versus the incident dimensionless water depth $k_{1} h_{1}$ for a different number of evanescent modes with a plane wave approximation solution. As shown in Case 2 in Figure 6, when there is no abrupt depth change and the evanescent modes are taken into account, only four evanescent modes are sufficient for the convergence, and the same suggestion of the evanescent modes for constant water depth can be found in Liu et al. [18]. However, when there is an abrupt depth change, velocity potential without evanescent modes cannot satisfy continuity or Laplace equation. Thus, the plane wave approximation solution (black dash line) drastically shows a different reflection coefficient from $N=4$ (black solid line), $N=16$ (black circle) results. Four evanescent modes for Case 3 show good results in the shallow water region $\left(k_{1} h_{1}<0.314\right)$ but not sufficient in the intermediate region $\left(k_{1} h_{1} \geq 0.314\right)$. Kirby and Dalrymple [4] reported that 16 evanescent modes provided adequate convergence for the trench problem.

Therefore, 27 steps of one side slope for the symmetric trench, double steps for the asymmetric trench, and 55 steps 


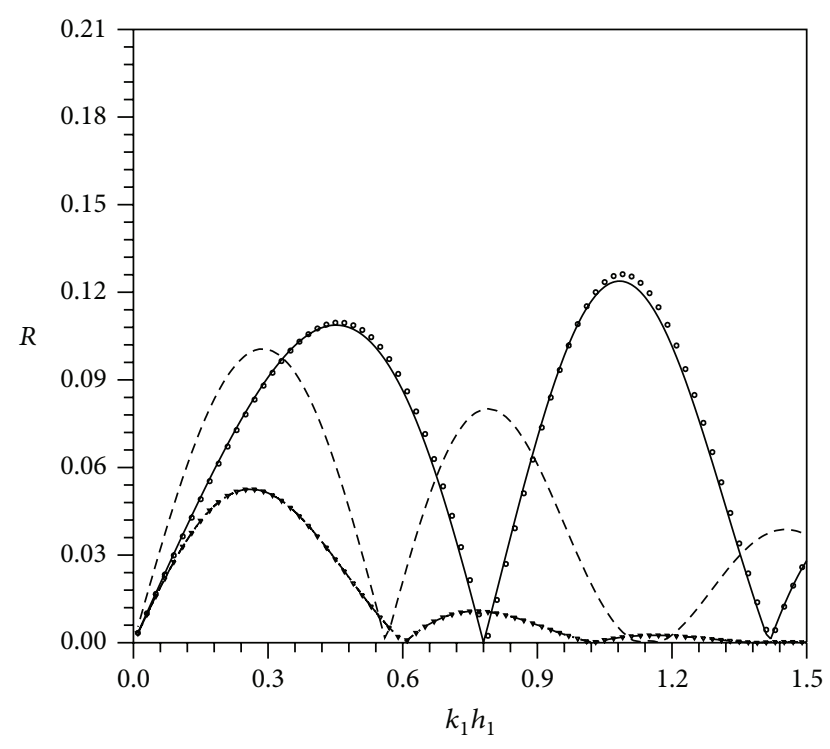

Case 2: $\quad-\cdots-$ Plane wave approximation $\ldots-N=4 \quad \cdots N=16$

Case 3: - - - Plane wave approximation $-N=4 \quad \ldots N=16$

FIgURE 6: A convergence test for different number of evanescent modes.

for sinusoidal shape shear current gave graphically precise results. Moreover, in order to take less time for computation, truncating up to 16 evanescent modes for an abrupt water depth change and 4 evanescent modes for constant water depth and following Cho and Lee [30], 4 evanescent modes for sinusoidally varying topography were used in the present calculations.

3.2. Effects of Shear Current Shape. A comparison of the results for various conditions was made in order to visualize the significance of the shear current shape (see Figure 7). For Case 1, a top-hat shear current, uniform water depth $\left(h_{1}=\right.$ $h_{\max }$ following the nomenclature in Figure 1), and uniform shear current $\left(\mathrm{Fr}_{\max }=0.1\right)$ were used. The water depth was fixed as $h_{\max }=2 h_{1}$ for Case 2 , a trench without the shear current, where the same result could be found in Liu et al. [18]. Case 3 is a combination of the trench and top-hat shear current. And Case 4 is the same trench as Case 3 but with a different shear current shape. In order to investigate the shear current shape effect, the sinusoidal shape shear current with maximum Froude number $\mathrm{Fr}_{\max }=0.15708$ calculated by $\left(1 / h_{1}\right) \int_{0}^{b} \operatorname{Fr}(x) d x$ was used in Case 4 to have the same cross section area of the shear current with Case 3.

As shown in Case 1 (line), although only a small amount of reflection occurs, the shear current could diffract the water wave in constant water depth. In Case 2 (dot), the reflection coefficients oscillate with the decreasing peaks and the transmission coefficients oscillate with increasing peaks as incident dimensionless water depth $k_{1} h_{1}$ increases. In Case 3 (dash line), the tendency of the peaks is similar to Case 2 , whereas the peaks and the complete transmission points change. Reflection coefficients and transmission coefficients at $k_{1} h_{1}=1.0$ taken from Liu et al. [18] show good agreement

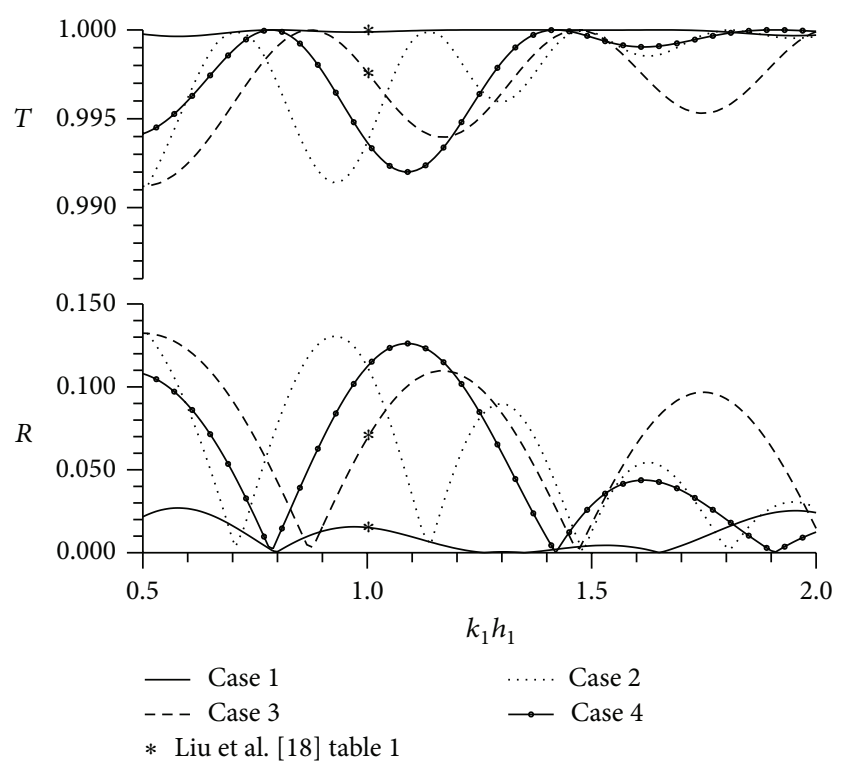

FIGURE 7: Comparison of reflection coefficients with transmission coefficients for various conditions.

TABLE 1: Maximum reflection coefficients with transmission coefficients, incident dimensionless water depth, and the conservation of the energy requirements.

\begin{tabular}{lcccc}
\hline Type & $k_{1} h_{1}$ & $R_{\max }$ & $T$ & $R_{\max }^{2}+\left(n_{j} k_{1} \cos \theta_{j} / n_{1} k_{j} \cos \theta_{1}\right) T^{2}$ \\
\hline Case 1 & 0.58 & 0.02698 & 0.99963 & 1.00000 \\
Case 2 & 0.40 & 0.14773 & 0.98903 & 1.00000 \\
Case 3 & 0.50 & 0.13239 & 0.99120 & 1.00000 \\
Case 4 & 1.09 & 0.12618 & 0.99201 & 1.00000 \\
\hline
\end{tabular}

with the present results of Cases 1 and 3. Comparing Case 3 with Case 4 (line with circle), the trend of reflection coefficients and transmission coefficients shows a completely different aspect. In Case 4, the first and third maximum reflection coefficients decrease while the second maximum reflection coefficient is enhanced. Thus, when solving the wave diffraction problem in the shear current affecting domain, it is important to apply the right shape of shear current even though it has small velocity.

The transmission coefficients and incident dimensionless water depth $k_{1} h_{1}$ when the reflection coefficients show maximum value are listed for each case in Table 1. The energy conservation relation obtained theoretically was checked to verify the reflection and transmission coefficients obtained from the numerical computation and also represented in Table 1. In this study, the reflection and transmission coefficients through all the incident dimensionless water depth $k_{1} h_{1}$ satisfy the conservation of the energy requirements.

3.3. Characteristics of Shear Current. In order to focus on the effect of the shear current, the water depth $h_{1}$ was set to constant. Several modifications were conducted to find the characteristics of the reflection coefficient due to the shear current. 


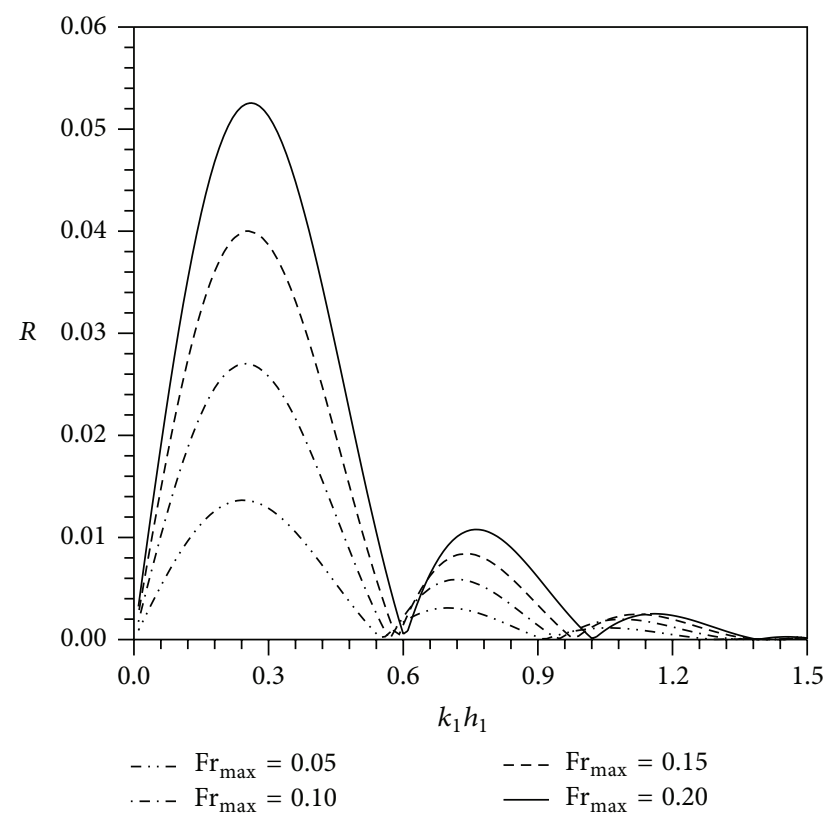

FIGURE 8: Reflection coefficients for constant water depth with different strength of shear current.

As shown in Figure 8, while the incident dimensionless water depth $k_{1} h_{1}$ values of zero reflection maintain almost the same positions, the maximum value of the reflection coefficient is proportional to the maximum Froude number $\mathrm{Fr}_{\max }$ when the incident angle and the width of shear current are fixed $\left(\theta=30^{\circ}, b / h_{1}=10\right)$. On the other hand, retaining the incident angle $\theta$ and the maximum Froude number $\mathrm{Fr}_{\max }$ to $30^{\circ}$ and 0.20 , Figure 9 shows that the maximum reflection coefficient shifts towards a small value of the incident dimensionless water depth $k_{1} h_{1}$ as the dimensionless width of shear current $b / h_{1}$ increases from 5 to 20 . If the cross section of the shear current calculated by $\left(1 / h_{1}\right) \int_{0}^{b} \operatorname{Fr}(x) d x$ is uniform, the first increasing slope of the reflection coefficient $R$ versus the incident dimensionless water depth $k_{1} h_{1}$ is equal until meeting the slightly small value of the maximum reflection coefficient as plotted in Figure 10. Figure 11 shows the reflection coefficients as the incident angle $\theta$ changes from $0^{\circ}$ to $45^{\circ}$. As discussed by McKee [25], zero reflection can be found in normally incident waves when the maximum Froude number $\mathrm{Fr}_{\max }$ and dimensionless width of shear current $b / h_{1}$ are fixed to 0.20 and 10 . The local maximum reflection coefficient occurs at $\theta \approx 27^{\circ}$. From this result, we can say that the main factor of the maximum reflection coefficient is the magnitude of shear current and the dimensionless width of the shear current determines the location of the maximum reflection coefficient.

3.4. Symmetric and Asymmetric Trapezoidal Trenches with Shear Current. In this subsection, both symmetric and asymmetric trenches were examined by changing the bottom width of a trench $b_{1} / h_{1}$ while keeping the top width, dimensionless depth of a trench $h_{\max } / h_{1}$, and the incident angle $\theta$ as 10,2 , and $30^{\circ}$ to find the optimal figure of a trench that could

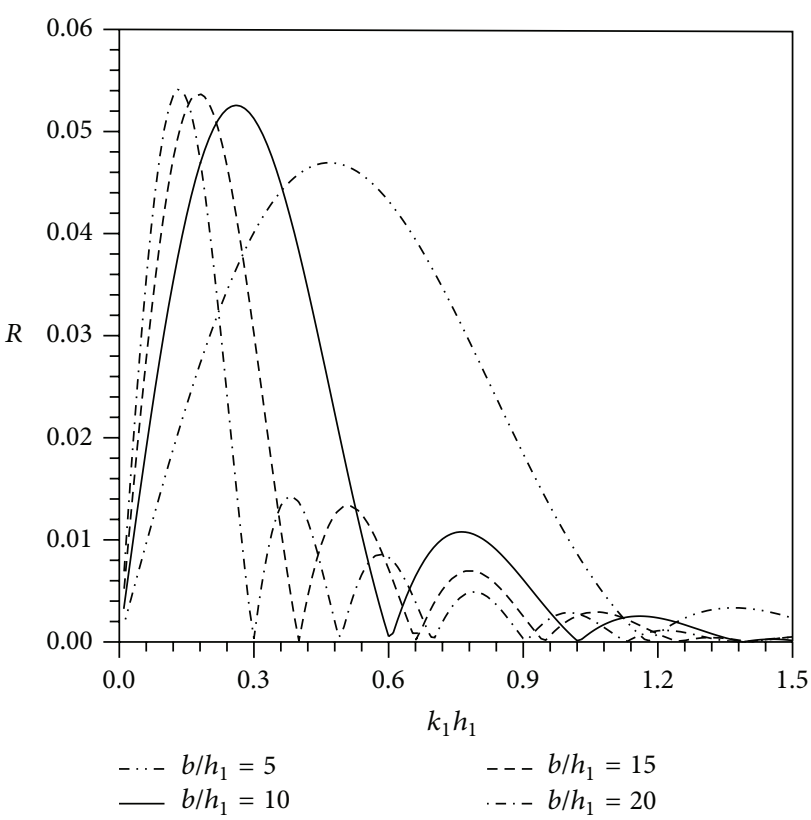

FIGURE 9: Reflection coefficients for constant water depth with different dimensionless width of shear current.

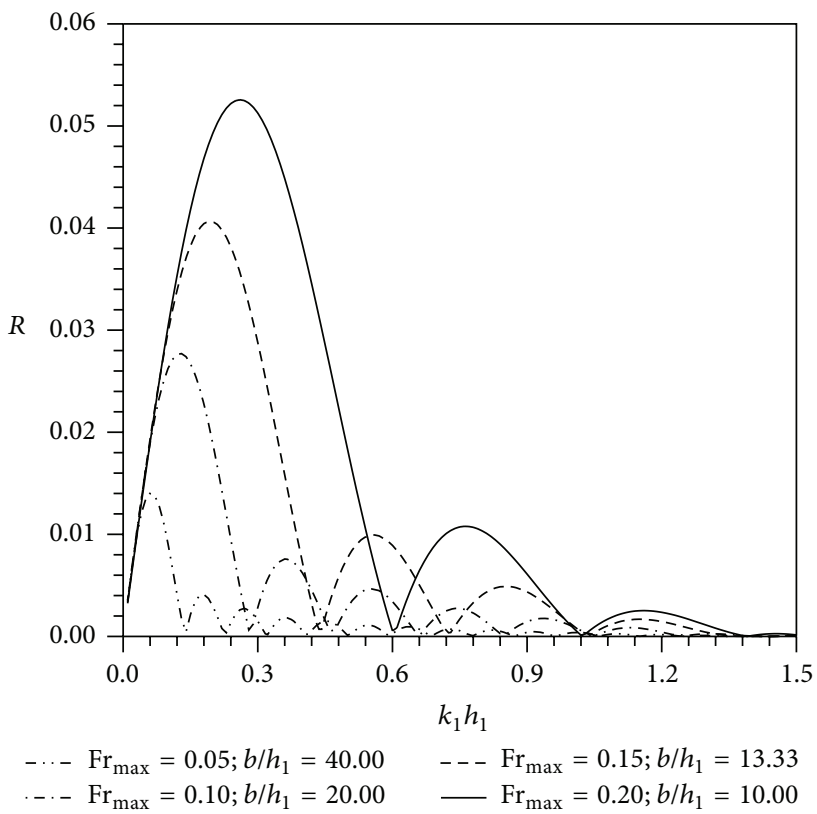

FIGURE 10: Reflection coefficients for constant water depth with different cross section of shear current.

reflect the water waves most highly when the shear current $\left(\mathrm{Fr}_{\max }=0.1\right)$ flows along the trench.

Figure 12 illustrates the reflection coefficients for different symmetric trench configurations. The reflection coefficients oscillate with decreasing peaks as the incident dimensionless water depth $k_{1} h_{1}$ increases. Increasing the transition slope (or the bottom width of trench $b_{1} / h_{1}$ ) is found to cause a high reflection coefficient. When the reflection coefficients near $k_{1} h_{1}=0.4$ are preserved, the reflection coefficients near 


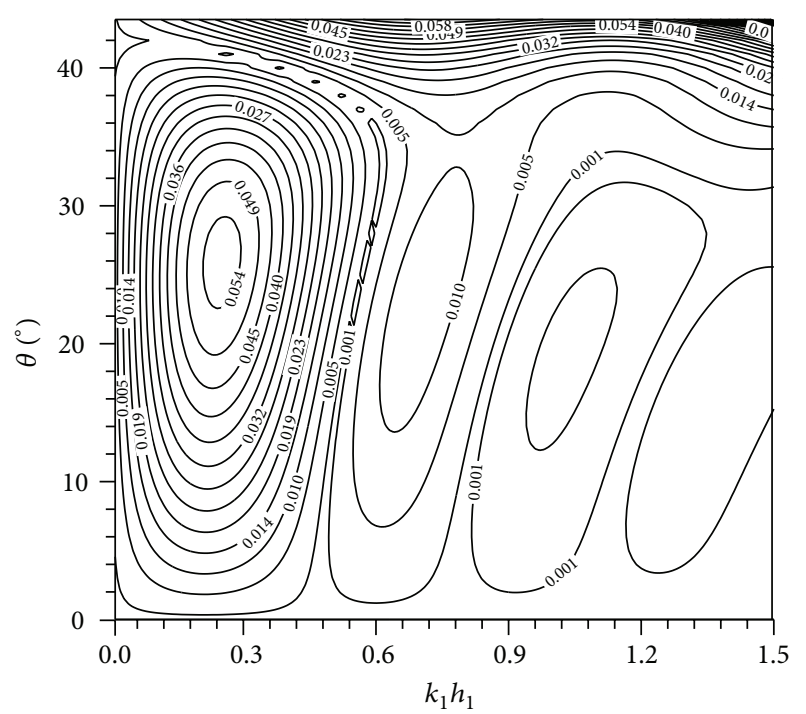

FIGURE 11: Reflection coefficients for constant water depth with a different incident angle.

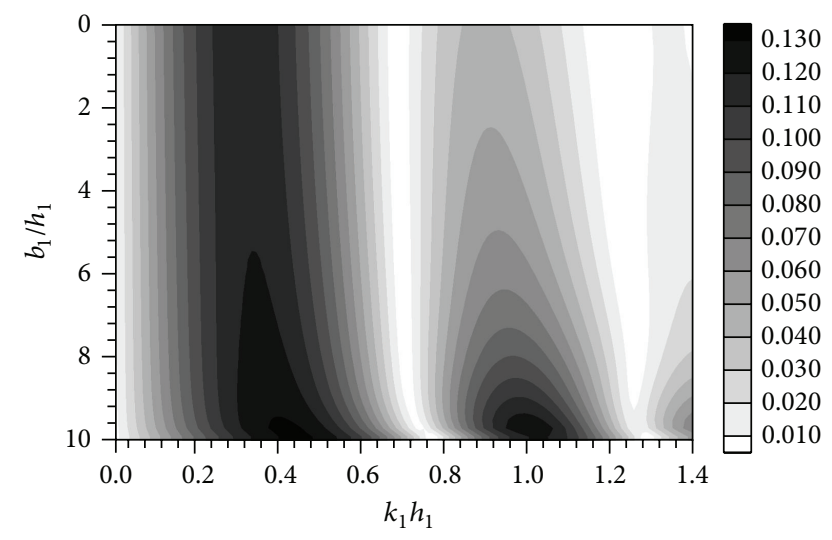

FIGURE 12: Reflection coefficients of a symmetric trapezoidal trench with shear current by sloped transitions.

$k_{1} h_{1}=1.0$ decrease rapidly as the bottom width of trench $b_{1} / h_{1}$ becomes narrow. The maximum reflection coefficient could be found at $k_{1} h_{1}=0.45, b_{1} / h_{1}=10$. Thus, the optimal figure of a symmetric trench is a rectangular shape where the top width and the bottom width of the trench are equal. This result agrees well with the trench without the shear current case of Bender and Dean [6].

Figure 13 shows the reflection coefficients for different asymmetric trench configurations for both the right (contour) and the left steep-slope trench (dashed line). The maximum reflection coefficient of the right and the left steep-slope trench could be found at the same condition with a symmetric trench. Bender and Dean [6] reported that the order of the transition slopes has no effect on the reflection coefficients when there is no shear current. However, when the shear current flows along the trench, the reflection coefficients of the left steep-slope trench are shown at the vicinities but slightly shifted than those of the right steep-slope trench.

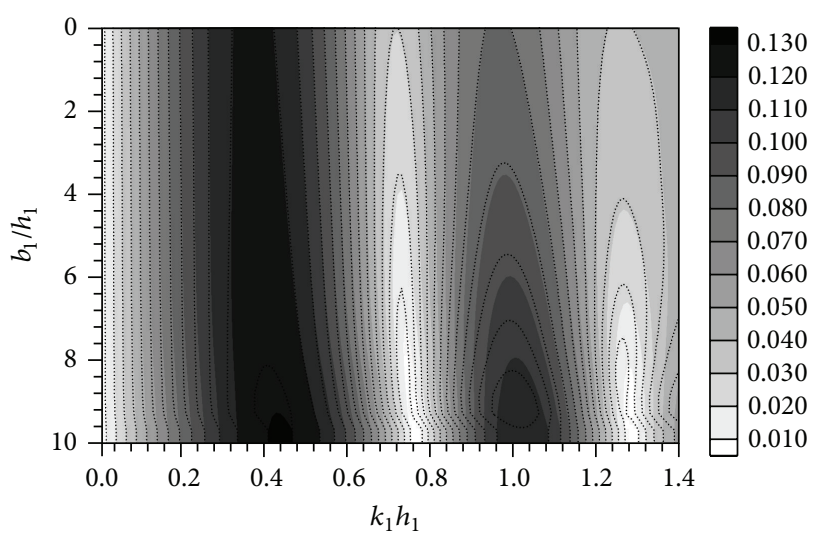

FIGURE 13: Reflection coefficients of an asymmetric trapezoidal trench with shear current by sloped transitions.

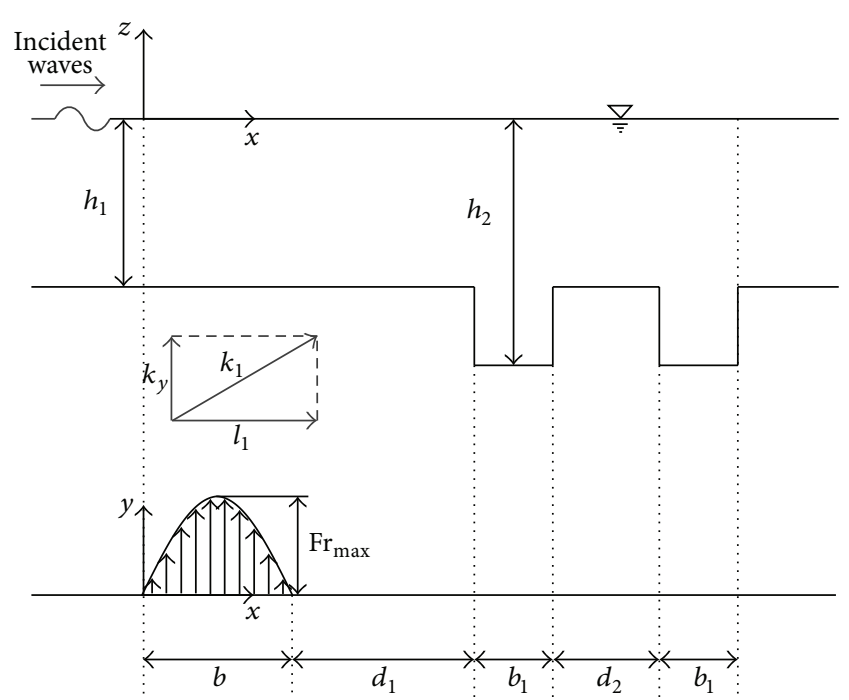

FIGURE 14: A schematic sketch of multiarrayed trenches with shear current.

This is due to the fact that the water waves passing the shear current experience changes in the phase speed and direction. Thus, when water waves have passed the right steep slope and shear current, the direction of water waves is different from the opposite case passing the left steep slope and shear current.

\section{Resonant Reflection due to Multiarrayed Trenches with Shear Current}

We have investigated the characteristic of shear current and found the optimal figure of trench. In this section, two cases are conducted to find the resonant reflection while using the optimal figure of a trench (see Figure 14) and sinusoidally varying topography with shear current (see Figure 15).

4.1. Multiarrayed Trenches with Shear Current. Mattioli [32] studied when the submerged breakwater arrays were in a 


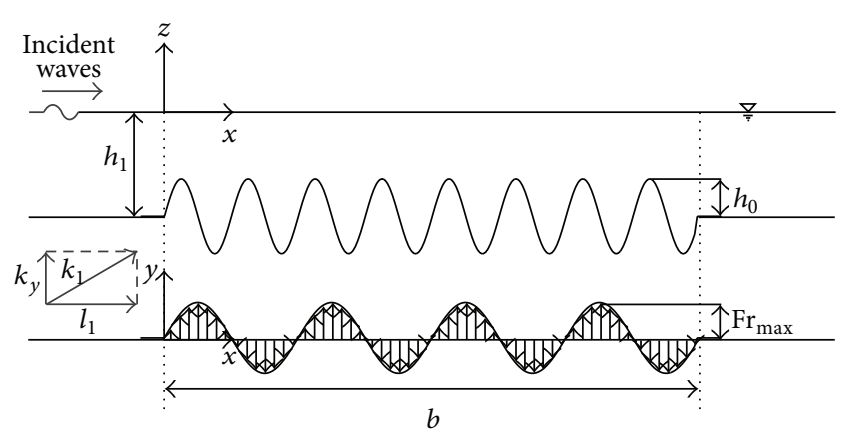

FIGURE 15: A schematic sketch of sinusoidally varying topography and sinusoidally varying shear current multiarrayed trenches with shear current.

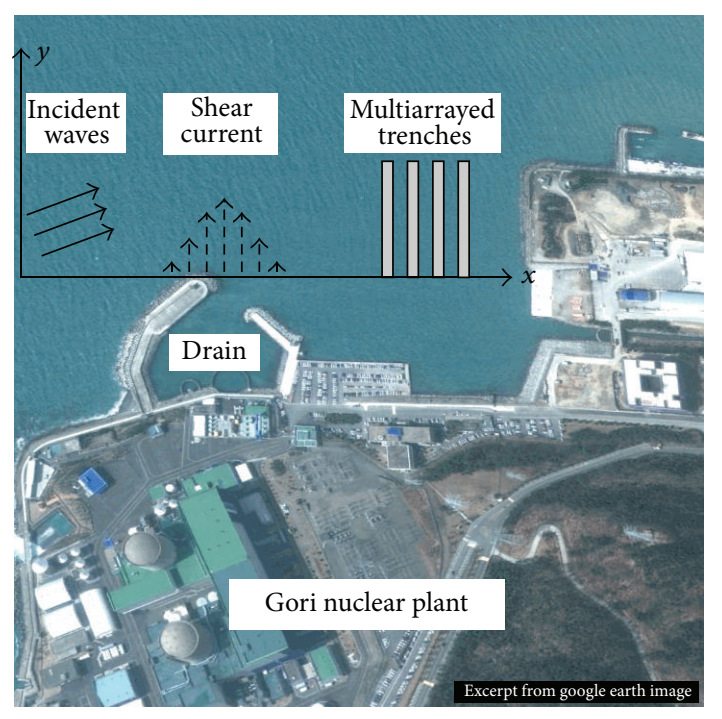

FIGURE 16: A target area image with a sketch diagram of multiarrayed trenches with shear current.

series whether the effects similar to the Bragg reflection would occur. When a water depth is required for waterways, trenches are more proper than submerged breakwaters. Most of the nuclear power plants in Korea are located in coastal region rather than in river region due to the high value of the coefficient of river regime that could limit the cooling water supply. The shear current caused by the drain of a nuclear plant could change the direction of the water waves coming to coastal region. Thus, when constructing trenches near the shear current affecting domain as shown in Figure 16, the interaction of the shear current and multiarrayed trenches should be investigated simultaneously. In this subsection, we studied the resonant reflection due to the idealized multiarrayed trenches configuration by taking the shear current into account.

When sinusoidal shape shear current $\left(\mathrm{Fr}_{\max }=0.2\right)$ flows antecedently, a comparison between the three cases characterized by the number of trenches was carried out. Figure 17 is a plot of the reflection coefficient as the dimensionless distance of trenches $d_{2} / h_{1}$ varies where $d_{2}$ is the distance

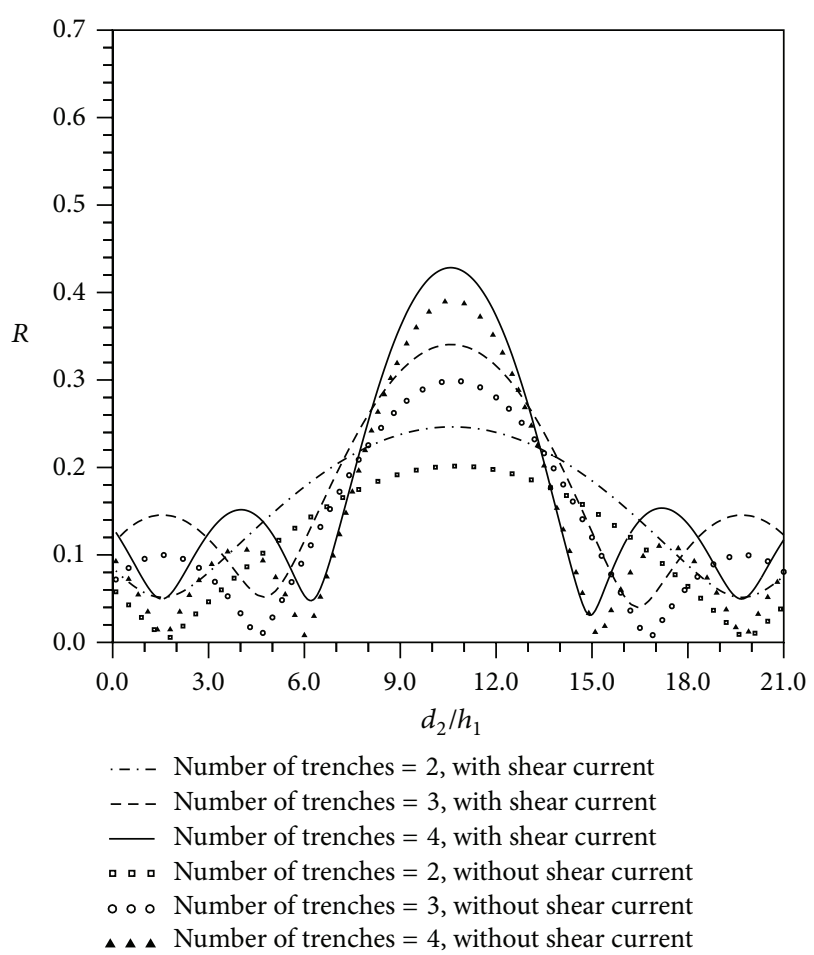

FIGURE 17: Reflection coefficients of multiarrayed trenches with shear current and without shear current.

between trenches. In order to investigate the effect of the shear current, we have plotted the results without the shear current. The optimal figure of the trench $\left(h_{\max } / h_{1}=1.5\right.$, $\left.b_{1} / h_{1}=10\right)$, the incident dimensionless water depth $\left(k_{1} h_{1}=\right.$ $0.2)$, the dimensionless distance between the shear current and trenches $\left(d_{1} / h_{1}=10\right)$, and the incident angle $(\theta=$ $30^{\circ}$ ) were kept fixed throughout the computations. When the shear current flows antecedently, the reflection coefficient increases by about 0.06 for all dimensionless distance of trenches $\left(d_{2} / h_{1}\right)$. It is clear that the number of trenches is associated with the maximum reflection coefficient and oscillating period of reflection coefficients. As the distance between trenches increases, the reflection coefficients oscillate and show the maximum value when the dimensionless distance of trenches $d_{2} / h_{1}$ is close to about 10.5.

In Figure 18, the reflection coefficients for the multiarrayed trenches in an optimal shape are shown without the shear current (black line) and with the shear current aside from the trenches (blue dashed line with rectangles). For both cases, decreasing local maximum reflection coefficients could be found as the incident dimensionless water depth $k_{1} h_{1}$ increases. The main discrepancy due to the shear current is changing the reflection coefficients in shallow water region $\left(k_{1} h_{1}<0.314\right)$ and deep water region $\left(k_{1} h_{1}>3.14\right)$. When considering the shear current, the local maximum reflection coefficients occur at the vicinities but a little small and slightly shifted toward left. This is not a general result that could be used for every multiarrayed trenches configurations; however, it should be noted that the shear current could affect the efficiency of multiarrayed trenches. 


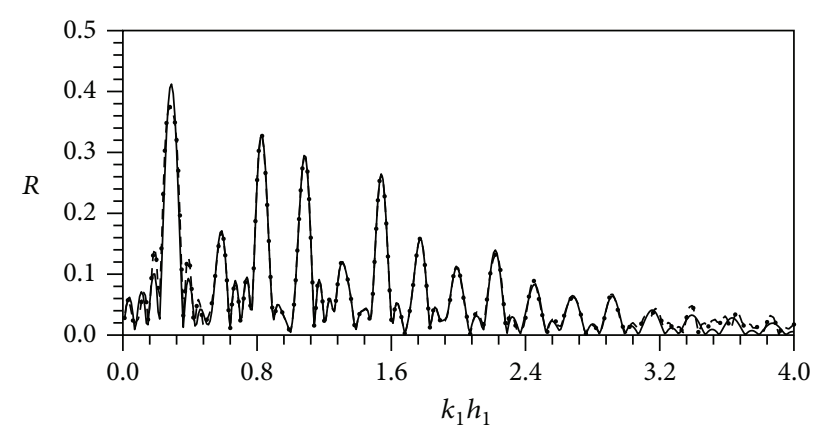

Without shear current

-.- With shear current

FIGURE 18: Comparison of reflection coefficients of multiarrayed trenches between with shear current and without shear current cases.

4.2. Sinusoidally Varying Topography and Shear Current. The shear current previously considered was unidirectional with a sinusoidal shape. However, the edge wave crossing the periodic rip current area or the complex region where the stream water and current flow in several directions cannot be interpreted in the unidirectional shear current. Thus, we idealized the bidirectional shear current as sinusoidally varying shape and employed the model to simulate the resonant reflection caused by sinusoidally varying topography and sinusoidally varying shear current. The water depth and the shear current are defined as shown in Table 2. $h_{0}$ is the amplitude of ripple and $l_{\text {topo }}, l_{\text {current }}$ are the wavenumber of the ripple and shear current, respectively.

Case 5 is of the propagation of water waves over corrugated bottoms without shear current $\left(\mathrm{Fr}_{\max }=0\right)$ and it was set up for the dimensionless amplitude of ripple $h_{0} / h_{1}=$ 0.32 , the wavenumber of ripple $l_{\text {topo }}=0.628$. Case 6 is the sinusoidally varying shear current flowing the constant water depth $\left(h_{0} / h_{1}=0\right)$, and the maximum Froude number $\mathrm{Fr}_{\max }=0.2$; the wavenumber of shear current $l_{\text {current }}=$ 0.314 was used. For both cases, the width of the topography and the shear current $b / h_{1}$ was 80 , and the incident angle $\theta$ was kept in $30^{\circ}$. Case 7 is the combination of Cases 5 and 6 where sinusoidally varying topography interacts with sinusoidally varying shear current.

In Figure 19, the reflection coefficients of the three cases are plotted as a function of the dimensionless wavenumber of topography $2 k / l_{\text {topo }}$. According to Dalrymple and Kirby [23], the resonant reflection of oblique incident water waves that pass sinusoidally varying topography occurs in the following condition:

$$
\frac{2 k_{1} \cos \theta}{l_{\text {topo }}}=n^{\prime}
$$

where $n^{\prime}$ is the order of resonant reflection. Case 5 (black circle) and Case 6 (white circle) are found to yield the same value as the Bragg reflection condition $\left(2 k / l_{\text {topo }} \approx 1.15\right.$, $2 k / l_{\text {current }} \approx 1.15$ ) based on the theoretical prediction. When the above cases are combined, that is, Case 7 (line), a double resonant reflection occurs. Also, the second order resonant
TABLE 2: Profiles of sinusoidally varying topography and sinusoidally varying topography shear current.

\begin{tabular}{lcc}
\hline Type & Water depth & Shear current \\
\hline Case 5 & $h(x)=h_{1}-h_{0} \sin \left(l_{\text {topo }} x\right)$ & $\operatorname{Fr}(x)=0$ \\
Case 6 & $h(x)=h_{1}$ & $\operatorname{Fr}(x)=\operatorname{Fr}_{\max } \sin \left(l_{\text {current }} x\right)$ \\
Case 7 & $h(x)=h_{1}-h_{0} \sin \left(l_{\text {topo }} x\right)$ & $\operatorname{Fr}(x)=\operatorname{Fr}_{\max } \sin \left(l_{\text {current }} x\right)$ \\
\hline
\end{tabular}

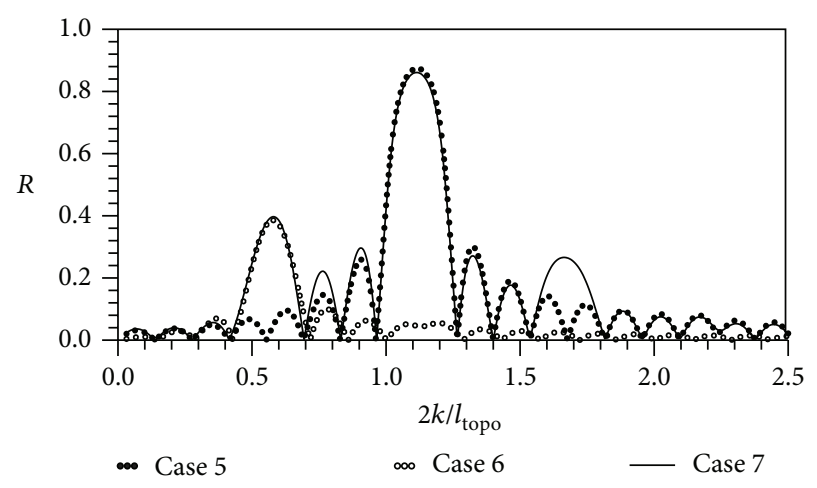

FIGURE 19: Reflection coefficients of sinusoidally varying topography and sinusoidally varying shear current.

reflection is enhanced by the interaction of sinusoidally varying topography and shear current. In Case 7, the sinusoidally varying shear current peak shifts, while the resonant reflection by the bottom corrugation moves little with limited reduction. This discrepancy could be thought of as the same reason as Figure 18 which showed a shift of the peaks with reduction caused by the shear current.

\section{Conclusions}

The eigenfunction expansion method model describing the diffraction of the monochromatic waves by the shear current at various topographies is presented. The model shows good agreement when checking the wave energy conservation and comparing the model with previous results. By the convergence test, 27 steps of one side slope for symmetric trench, double steps for asymmetric trench, and 55 steps for sinusoidal shape shear current are suggested for steps. And 16 evanescent modes for abrupt water depth change, 4 evanescent modes for constant water depth, and sinusoidally varying topography are suggested for evanescent modes.

Several new results were found in this study: The maximum reflection coefficient is proportional to the magnitude of the shear current, and its location is mainly affected by the dimensionless width of the shear current. When the shear current condition is fixed, an orthogonal sloped trench shows the maximum reflection coefficient. The resonant reflection was checked by multiarrayed trenches with shear current. By superposing sinusoidally varying topography and sinusoidally varying shear current that have different wavenumbers, double resonant reflection occurs and the second order resonant reflection is enhanced. 
Because the shear current could shift and change the aspect of the reflection coefficients, shear current should not be omitted while investigating the wave diffraction problem. Hence, the result of this study could provide a possible means for designing counter facilities to protect coastal structures from wave attacks by reflecting the wave energy, especially when the shear current plays a role.

\section{Conflict of Interests}

The authors declare that they have no conflict of interests regarding the publication of this paper.

\section{Acknowledgments}

This research was supported by the Basic Science Research Program through the National Research Foundation of Korea funded by the Ministry of Education, Science and Technology (2010-0022337).

\section{References}

[1] C. C. Mei, The Applied Dynamics of Ocean Surface Waves, World Scientific, Singapore, 1989.

[2] K. Takano, "Effets d'un obstacle parallélépipédique sur la propagation de la houle," La Houille Blanche, vol. 15, pp. 247$267,1960$.

[3] J. W. Miles, "Surface-wave scattering matrix for a shelf," Journal of Fluid Mechanics, vol. 28, no. 4, pp. 755-767, 1967.

[4] J. T. Kirby and R. A. Dalrymple, "Propagation of obliquely incident water waves over a trench," Journal of Fluid Mechanics, vol. 133, pp. 47-63, 1983.

[5] J. B. Lassiter III, The propagation of water waves over sediment pockets [Ph.D. thesis], 1972.

[6] C. J. Bender and R. G. Dean, "Wave transformation by twodimensional bathymetric anomalies with sloped transitions," Coastal Engineering, vol. 50, no. 1-2, pp. 61-84, 2003.

[7] R. Chakraborty and B. N. Mandal, "Water wave scattering by a rectangular trench," Journal of Engineering Mathematics, vol. 89, no. 1, pp. 101-112, 2014.

[8] J.-J. Xie, H.-W. Liu, and P. Lin, "Analytical solution for long-wave reflection by a rectangular obstacle with two scour trenches," Journal of Engineering Mechanics, vol. 137, no. 12, pp. 919-930, 2012.

[9] S. Gangopadhyay and U. Basu, "Scattering of capillary waves in front of a semi-infinite dock in an ocean with porous undulatory bottom," International Journal of Scientific \& Technology Research, vol. 2, no. 1, 2013.

[10] I. H. Cho and M. H. Kim, "Transmission of oblique incident waves by a submerged horizontal porous plate," Ocean Engineering, vol. 61, pp. 56-65, 2013.

[11] Q. Lin and D. Q. Lu, "Water wave diffraction by a bottommounted circular cylinder clamped to an elastic plate floating on a two-layer fluid," European Journal of Mechanics B: Fluids, vol. 44, pp. 10-21, 2014.

[12] S.-C. Jiang, Y. Gou, B. Teng, and D.-Z. Ning, "Analytical solution of a wave diffraction problem on a submerged cylinder," Journal of Engineering Mechanics, vol. 140, no. 1, pp. 225-232, 2014.
[13] R. D. MacIver, R. R. Simons, and G. P. Thomas, "Gravity waves interacting with a narrow jet-like current," Journal of Geophysical Research C: Oceans, vol. 111, no. 3, 2006.

[14] D. V. Evans, "The transmission of deep-water waves across a vortex sheet," Journal of Fluid Mechanics, vol. 68, no. 2, pp. 389401, 1975.

[15] J. Smith, "On surface gravity waves crossing weak current jets," Journal of Fluid Mechanics, vol. 134, pp. 277-299, 1983.

[16] J. Smith, "On surface waves crossing a step with horizontal shear," Journal of Fluid Mechanics, vol. 175, pp. 395-412, 1987.

[17] J. T. Kirby, R. A. Dalrymple, and S. N. Seo, "Propagation of obliquely incident water-waves over a trench .2. Currents flowing along the trench," Journal of Fluid Mechanics, vol. 176, pp. 95-116, 1987.

[18] P. L.-F. Liu, Y.-S. Cho, J. K. Kostense, and M. W. Dingemans, "Propagation and trapping of obliquely incident wave groups over a trench with currents," Applied Ocean Research, vol. 14, no. 3, pp. 201-213, 1992.

[19] W. D. McKee, "The propagation of water waves across a laterally sheared current," Applied Ocean Research, vol. 28, no. 5, pp. 339344, 2006.

[20] W. D. McKee, "Water wave propagation across a shearing current," Wave Motion, vol. 9, no. 3, pp. 209-215, 1987.

[21] W. D. Mckee, "A model for surface wave propagation across a shearing current," Journal of Physical Oceanography, vol. 26, no. 2, pp. 276-278, 1996.

[22] A. G. Davies and A. D. Heathershaw, "Surface-wave propagation over sinusoidally varying topography," Journal of Fluid Mechanics, vol. 144, pp. 419-443, 1984.

[23] R. A. Dalrymple and J. T. Kirby, "Water waves over ripples," Journal of Waterway, Port, Coastal and Ocean Engineering, vol. 112, no. 2, pp. 309-319, 1986.

[24] E. Guazzelli, V. Rey, and M. Belzons, "Higher-order Bragg reflection of gravity surface waves by periodic beds," Journal of Fluid Mechanics, vol. 245, pp. 301-317, 1992.

[25] W. D. McKee, "Reflection of water waves by a weak rapidly varying shearing current," Wave Motion, vol. 20, no. 2, pp. 143149, 1994

[26] Y.-S. Cho and C. Lee, "Calculation of reflection and transmission coefficients of waves over a varying topography," Journal of Korean Society of Civil Engineers, vol. 18, no. 1-4, pp. 351-358, 1998.

[27] J. T. Kirby and J. P. Anton, "Bragg reflection of waves by artificial bars," Coastal Engineering Proceedings, vol. 1, no. 22, 1990.

[28] T.-W. Hsu, L.-H. Tsai, and Y.-T. Huang, "Bragg scattering of water waves by multiply composite artificial bars," Coastal Engineering Journal, vol. 45, no. 2, pp. 235-253, 2003.

[29] J.-M. Kim, T.-H. Jung, J.-W. Lee, and Y.-S. Cho, "Reflection of directional random waves propagating over various shapes of trench," in Proceedings of the 19th International Society of Offshore and Polar Engineers Conference (ISOPE OMS '09), pp. 1174-1179, June 2009.

[30] Y.-S. Cho and C. Lee, "Resonant reflection of waves over sinusoidally varying topographies," Journal of Coastal Research, vol. 16, no. 3, pp. 870-876, 2000.

[31] K. Belibassakis, "Propagation of water waves through shearing currents in general bathymetry," in Maritime Transportation and Exploitation of Ocean and Coastal Resources, Two Volume Set, pp. 981-989, Taylor \& Francis, 2005.

[32] F. Mattioli, "Resonant reflection of a series of submerged breakwaters," Il Nuovo Cimento C, vol. 13, no. 5, pp. 823-833, 1990. 


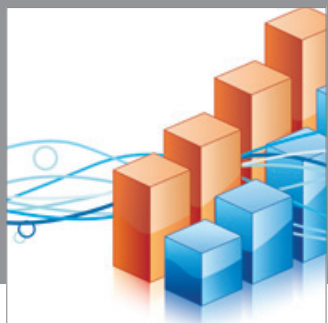

Advances in

Operations Research

mansans

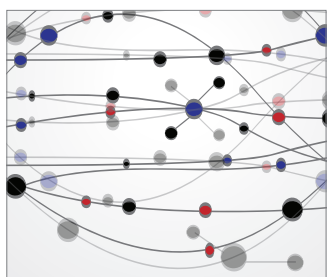

The Scientific World Journal
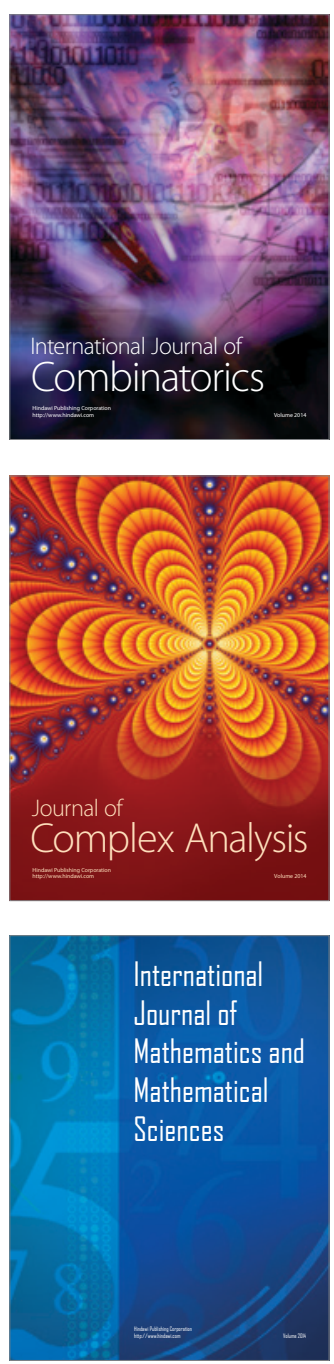
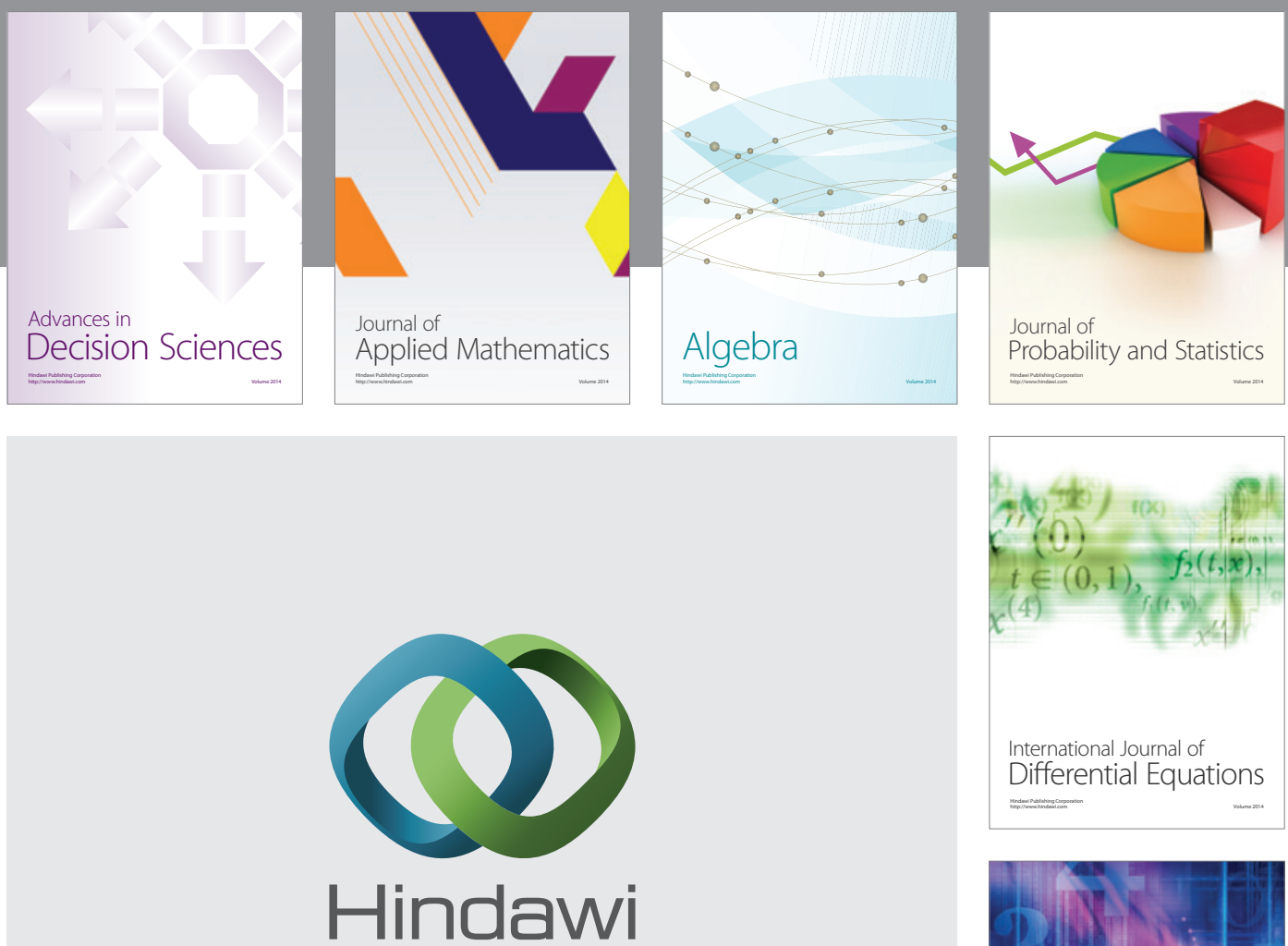

Submit your manuscripts at http://www.hindawi.com
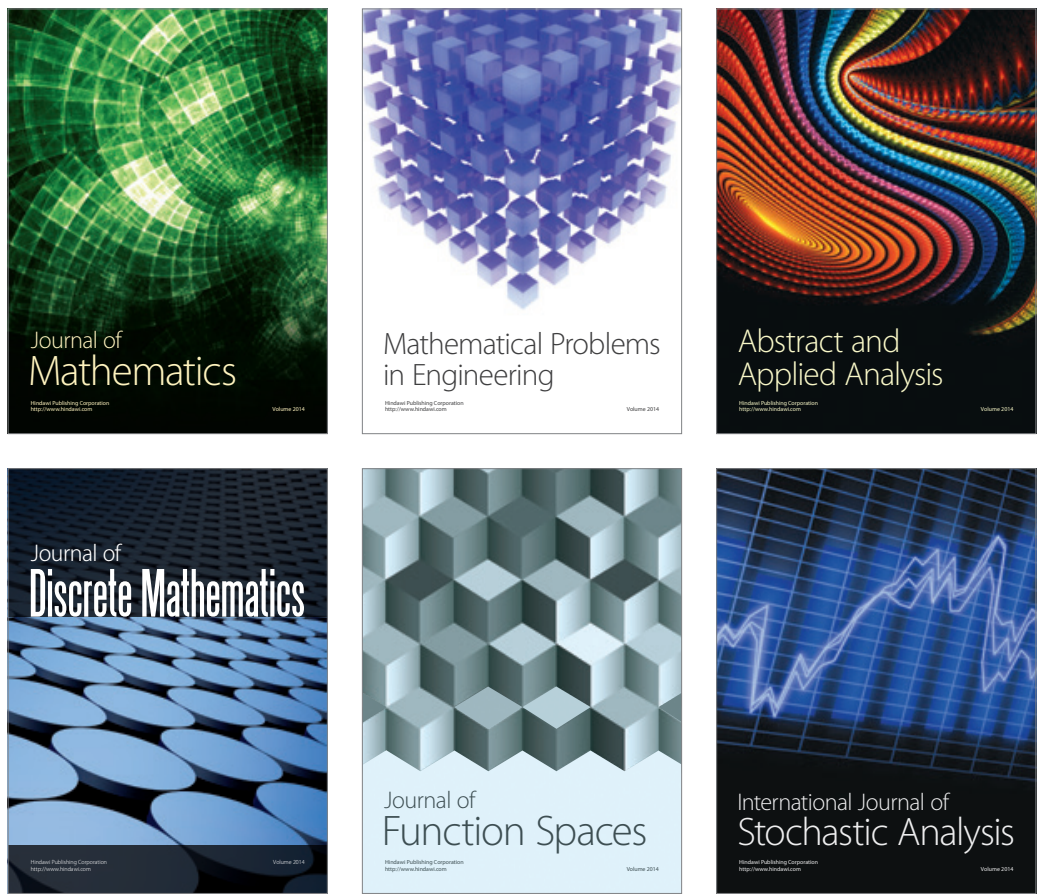

Journal of

Function Spaces

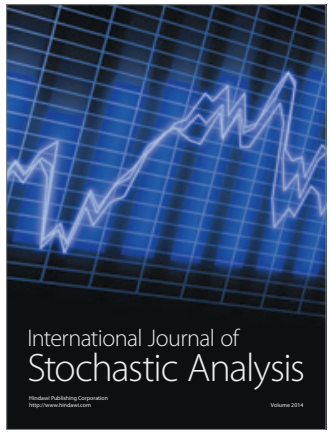

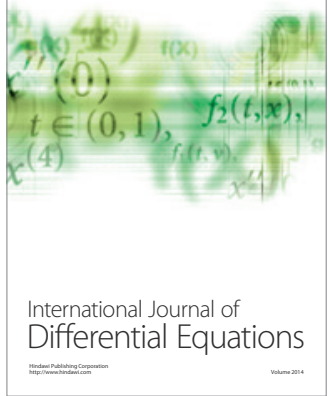
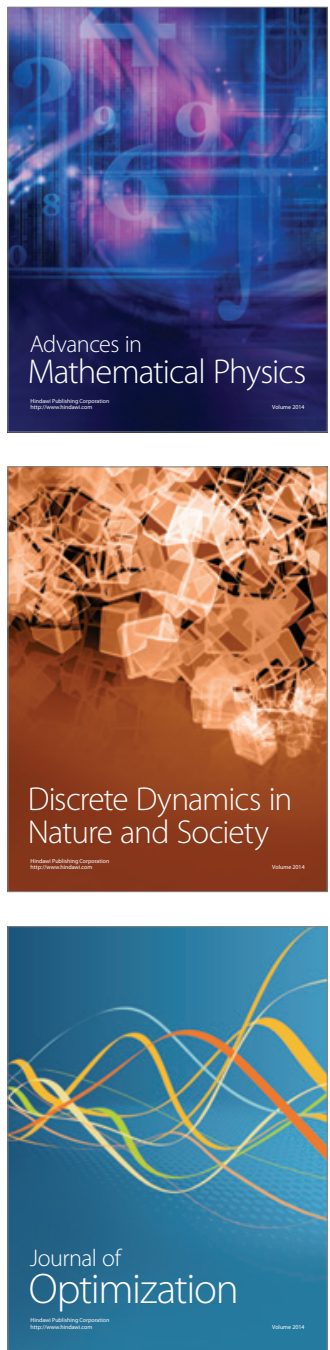